\title{
Prefrontal cortex and the mediation of proactive interference in working memory
}

\author{
BRADLEY R. POSTLE, LAUREN N. BRUSH, and ANDREW M. NICK \\ University of Wisconsin, Madison, Wisconsin
}

\begin{abstract}
Mediating proactive interference (PI), the deleterious effect of antecedent information on current memory representations, is believed to be a key function of prefrontal cortex (PFC). Item-specific PI results when an invalid probe matches a memorandum from the preceding trial; item-nonspecific PI is produced by the accumulation of no-longer-relevant items from previous trials. We tested the hypothesis that these two types of PI are mediated by common PFC-based processes with an fMRI study of a delayed-recognition task designed to produce both types of PI. Our results indicated that the fMRI correlates of both effects were restricted both to Brodmann's area 45 in the left hemisphere and to the memory probe epoch of the trial. These results suggest that a unification of the literatures and approaches that have independently studied these phenomena might offer a fruitful new perspective from which to study the relations between working memory, executive control, and the PFC.
\end{abstract}

Prominent in the legacy of Patricia Goldman-Rakic is an appreciation for the powerful insights that physiological data bring to investigations of prefrontal cortex (PFC) contributions to working memory function. In her wake, a next generation of systems and cognitive neuroscientists seeks to advance our understanding of the complex factors relating PFC function, working memory, and the mind (Goldman-Rakic, 1992). Our own efforts in this area have been motivated by the view that working memory storage functions are not supported by PFC, but rather in a domain-specific manner by brain systems that serve sensory-, representation-, and action-related functions (e.g., Postle, Awh, Jonides, Smith, \& D'Esposito, 2004; Postle, Berger, \& D'Esposito, 1999; Postle \& D'Esposito, 1999, 2003; Postle, Druzgal, \& D’Esposito, 2003). PFC, in contrast, implements control, including the protection of the contents of working memory from the disruptive effects of interference (e.g., Knight, Staines, Swick, \& Chao, 1999; Postle \& Brush, 2004). The present article is focused on the mediation of proactive interference (PI), the deleterious effect of previously remembered information on current memory representations.

PI derives from two sources. Item-specific PI occurs when an invalid probe matches a memorandum from the preceding trial (Monsell, 1978). Item-nonspecific PI is produced by the accumulation of no-longer-relevant items that have been remembered on previous trials. The for-

This research was supported by National Institutes of Health Grant MH064498. We thank Olufunsho Faseyitan, Christopher Jordan, and Terrence Oakes for assistance with data collection and analysis and figure production, and John Ollinger, Steven Petersen, and Kristina Visscher for helpful discussions of the global signal results. Correspondence relating to this article may be sent to B. R. Postle, Department of Psychology, University of Wisconsin, 1202 W. Johnson Street, Madison, WI 53706 (e-mail: postle@wisc.edu). mer source has been well characterized behaviorally and neurally. It is associated with longer reaction times (RT) and greater activity in the memory probe/response epoch of the trial in Brodmann's area (BA) 45 of the left inferior PFC (D’Esposito, Postle, Jonides, \& Smith, 1999; Jonides, Badre, Curtis, Thompson-Schill, \& Smith, 2002; Jonides, Marshuetz, Smith, Reuter-Lorenz, \& Koeppe, 2000; Jonides, Smith, Marshuetz, Koeppe, \& ReuterLorenz, 1998; Nelson, Reuter-Lorenz, Sylvester, Jonides, \& Smith, 2003). Item-nonspecific PI, in turn, has long been understood to be an important factor in forgetting from short-term and working memory (Hasher \& Zacks, 1988; Keppel \& Underwood, 1962; May, Hasher, \& Kane, 1999; Wickens, Born, \& Allen, 1963), but little is known about its neural bases. The purpose of the present study was to test the prediction that the neural correlates of these two types of PI share common temporal dynamics and topology. It was motivated by the hypothesis that the effects of item-specific and item-nonspecific PI on working memory may be mediated by a common process or set of processes (Postle \& Brush, 2004).

There is considerable evidence that item-specific PI is mediated by a control process (and, therefore, that its neurophysiological correlates aren't simply duty-cycle effects attributable to longer RTs): The behavioral effect of item-specific PI is small and its neurophysiological correlate robust in healthy young adults (Jonides et al., 1998), but the converse is true in the elderly (Jonides et al., 2000); also, a patient with a lesion encompassing left BA 45 is disproportionately sensitive to item-specific PI compared with PFC-lesioned control subjects in whom BA 45 is unaffected (Thompson-Schill et al., 2002). Thus, one theoretical implication of the common-processes hypothesis is that it lends support to the idea that the agerelated vulnerability of working memory performance to item-nonspecific PI (May et al., 1999) is attributable to 
a weakening control mechanism (e.g., inhibition; Hasher \& Zacks, 1988; Lustig, May, \& Hasher, 2001). The hypothesis supports this idea by simultaneously suggesting a common underlying mechanism for the mediation of item-specific and nonspecific PI and ruling out the alternative explanation that the increased age-related sensitivity to item-nonspecific PI is attributable to inefficient encoding. (These issues are discussed in detail in Postle \& Brush, 2004.) Finally, it is worth noting that a common-processes assumption is not necessarily the default assumption for all types of interference. For example, Nelson et al. (2003) have recently demonstrated the dissociability, within frontal cortex, of effects related to item-specific PI (localized to left inferior PFC) and response selection [localized to medial superior frontal cortex and anterior cingulate cortex (ACC)].

As a first step toward evaluating the common-processes hypothesis, we have begun by investigating whether the two types of PI evoke neural effects in similar brain areas and with similar time courses. The first criterion draws on the rationale that a necessary, but not sufficient, criterion for a common-processes explanation of any two cognitive phenomena is that the same brain region be sensitive to manipulation of both. ${ }^{1}$ With regard to temporal dynamics, the neural correlate of item-specific PI is phasic and isolated to the probe epoch of delayed-recognition trials (D’Esposito et al., 1999; Postle, Berger, Goldstein, Curtis, \& D'Esposito, 2001). The temporal dynamics of item-nonspecific PI, however, are still poorly understood. A previous study revealed a clear probe-related effect, but was inconclusive as to whether other epochs of the trial may have also been sensitive to item-nonspecific PI (Postle \& Brush, 2004). A common mechanism could, in principle, operate either phasically or tonically to mediate item-nonspecific PI but operate only phasically to mediate the effects of item-specific PI. Interestingly, it has been proposed that control processes with both temporal profiles are supported by left inferior PFC (Braver, Gray, \& Burgess, in press; Reynolds, Donaldson, Wagner, \& Braver, 2004), the region of primary interest in the present study.

In an initial study, we reanalyzed the behavioral and fMRI results from seven independent data sets by recoding the data as a function of trial position within the block. The results indicated that both the portion of left anterior inferior frontal gyrus corresponding to BA 45 and the left dorsolateral PFC are differentially sensitive to the build-up of item-nonspecific PI (Postle \& Brush, 2004). One noteworthy feature of the results was a decline in the probe- (but not delay-) evoked global signal as a function of trial position, a decline that affected all regions of PFC and ACC except for left BA 45 and left dorsolateral PFC. (The global signal is the blood oxygen level dependent signal pooled across every voxel of the brain, as though the entire brain comprised one enormous voxel.) The trend in the two left hemisphere regions was flat instead of negative, and we speculated that this difference may have reflected a positive-trending ef- fect of item-nonspecific PI superimposed on a negativegoing global signal. These results established the plausibility of the common-processes account.

The aim of the present study was to apply a more stringent test of the common-processes hypothesis by assessing the effects of item-specific and nonspecific PI within the same subjects, during the same experiment. To do this, we embedded several "recent negative" (RN; i.e., item-specific PI-producing) memory probes within each block of trials and then analyzed the fMRI data in two ways, by recency of negative probes and by trial position (to evaluate item-nonspecific PI). In order to maximize the release from PI (Wickens, 1973; Wickens et al., 1963) between blocks of trials, and thereby to maximize our sensitivity to item-nonspecific PI, stimulus domain varied from block to block. (Because of this, our design also permitted us to examine questions of stimulus-domain specificity in the mediation of PI.) We predicted that of the PFC and ACC regions that we would examine, only left BA 45 would demonstrate sensitivity to both itemspecific and nonspecific PI.

\section{METHOD}

\section{Subjects}

Twenty-five members of the University of Wisconsin-Madison community ( 16 female; mean age of all subjects $=22.2$ years, $S D=3.1$ ) participated. None reported any medical, neurological, or psychiatric illness or taking any prescription medication. All were right-handed, and all gave informed consent.

\section{Materials}

The experiment comprised seven blocks of 16 delayed-recognition trials. Each block featured stimuli from a different domain, and the composition of each trial was determined by randomly selecting stimuli (targets and probes) without replacement from pools of between 10 and 21 items: 21 consonant letters; 10 color patches (white, gray, red, pink, orange, yellow, green, light blue, indigo, and purple); 16 locations; 16 of the 19 highest frequency names of flowers $^{2}$ (Battig \& Montague, 1969); the digits "0" through " 9 "; 16 male faces; and 16 silhouette polygon shapes (Attneave \& Arnoult, 1956) determined to be difficult to verbalize (Vanderplas \& Garvin, 1959). Either one or four items (one face or shape; four colors, digits, words, locations, or letters) were presented as memoranda on each trial. Four-item target sets (apart from the locations task) were arrayed about a fixation cross so that they formed a square around it. All stimuli were white (apart from the color patches and grayscale faces) and were presented against a black background.

There were three forms of the experiment, each featuring a different block order, and each block (both within and across forms) featuring a different randomly determined order of stimuli. Block order was pseudorandomized in each of the three forms of the experiment, with the constraint that no two blocks of overtly verbalizable material (i.e., words, digits, and letters) were presented consecutively. At this intermediate point of block construction, each of the 16 trials in a block was invalid, because final probes were not yet selected for the targets. Next, the order of trial types within each block was determined by selecting randomly (without replacement) from a pool of seven nonrecent positive (NP), four nonrecent negative (NN: producing no item-specific PI), and five recent negative (RN: producing item-specific PI) probes. The probes of the $7 \mathrm{NP}$ trials were then changed, and selected randomly in the case of the four-item trials. Finally, some changes were made to the original 
randomly determined order of memoranda, so that $\mathrm{RN}$ probes were preceded by a matching memorandum (i.e., a lure) on the two preceding trials, and NP and NN probes were preceded by no matching memoranda on the two preceding trials. (Exceptions to the lures-on-two-preceding-trials rule were stimulus domains for which only one memorandum was presented per trial and instances when the second trial in a block was an RN trial. In these cases, RN probes were only preceded by a lure on the previous trial.)

\section{Procedure}

The stimulus domain for each block was identified in instructions that were presented prior to the beginning of each block. All trials lasted $8 \mathrm{sec}$ from target onset to probe onset, with an intertrial interval of $13 \mathrm{sec}$. Targets were presented for $1,000 \mathrm{msec}$, with the exception that words were presented for $1,500 \mathrm{msec}$. Subjects were trained to remember the target item(s) across the delay period and to respond to the probe with a "yes" (right button) or "no" (left button) thumbpress. They were instructed to respond to the probe as quickly and accurately as possible. Subjects were assigned to a form of the experiment based on recruitment order, such that the first and fourth subjects were tested with Form 1, the second and fifth subjects with Form 2, and so on.

\section{fMRI Data Acquisition and Preprocessing}

Whole-brain images were acquired with a 3.0T scanner (GE Signa VH/i). High-resolution T1-weighted images (30 axial slices, $0.9375 \times 0.9375 \times 4 \mathrm{~mm}$ ) were obtained in all subjects, and a gradient echo, echoplanar sequence $(\mathrm{TR}=2,000 \mathrm{msec}, \mathrm{TE}=50 \mathrm{msec})$ was used to acquire data sensitive to the blood oxygen level dependent (BOLD) signal (Kwong et al., 1992; Ogawa et al., 1992) within a $64 \times 64$ matrix ( 30 axial slices coplanar with the T1 acquisition, $3.75 \times 3.75 \times 4 \mathrm{~mm})$. Scans of the delayed-recognition task were preceded by a scan in which we derived an estimate of the hemodynamic response function (HRF) for each subject. During this scan, each subject performed a simple reaction-time task that required a bimanual buttonpress once every $20 \mathrm{sec}$ in response to a brief change in shape of the fixation stimulus. A partial $F$ test associated with a Fourier basis covariate set (Josephs, Turner, \& Friston, 1997) was used to evaluate the significance of task correlated activity in each voxel of primary somatosensory and motor cortical regions of interest (ROIs). An HRF estimate was extracted from the suprathreshold voxels of these ROIs by spatially averaging their time series, filtering the resultant averaged fMRI time series to remove high $(>0.244 \mathrm{~Hz})$ and low $(<0.05 \mathrm{~Hz})$ frequencies, adjusting the estimate to remove the effects of nuisance covariates (Friston, Holmes, Poline, Heather, \& Frackowiak, 1995), and trial averaging. The HRF characterizes the fMRI response resulting from a brief impulse of neural activity (Boynton, Engel, Glover, \& Heeger, 1996) and can vary markedly across subjects (Aguirre, Zarahn, \& D'Esposito, 1998; Handwerker, Ollinger, \& D'Esposito, 2004). The subject-specific HRFs were used to convolve independent variables entered into the modified general linear model (GLM; Worsley \& Friston, 1995) that we used to analyze the data from the scans of the working memory task. The seven scans of the working memory task each lasted $6 \mathrm{~min} 12 \mathrm{sec}(5 \mathrm{~min} 52 \mathrm{sec}$ of task preceded by $20 \mathrm{sec}$ of dummy pulses to achieve a steady state of tissue magnetization).

\section{Analyses}

The primary goal of this experiment was to compare the neural correlates of item-specific and item-nonspecific PI. In order to accomplish this, the data were coded in two different ways: For itemnonspecific PI, data were coded according to a trial's position within its block, collapsing across blocks. In this way, we sought to detect a trend that evolved across time, so that the effect of the trend for any one trial would be a function of the number of antecedent trials in the block. For item-specific PI, data were coded according to the three trial types- $\mathrm{NP}, \mathrm{NN}$, and $\mathrm{RN}$ - again collapsed across blocks.

\section{Behavioral Data}

Item-nonspecific PI. The RT associated with each stimulus domain was first mean-normalized in order to remove any offsets in RT that could be attributed to the difficulty or some other factor specific to that domain. Reaction times were then collapsed across blocks, and the mean RT at each trial position was computed. Based on our previous study (Postle \& Brush, 2004), we predicted that there would be a significant positive linear relationship between RT and trial position for the first five trials of each block- a behavioral correlate of the build-up of item-nonspecific PI. We also predicted that this PI effect would saturate after the fifth trial, with the trend of increasing RTs plateauing and variability in RT becoming larger for the 6 th through 16 th trials than for the first 5 . Thus, our principal variable of interest was the slope of RT across the first 5 trials, and particularly whether it differed from zero. We implemented this test using a multivariate analysis of variance (MANOVA) rather than a regression or repeated measures ANOVA because of MANOVA's less restrictive assumptions and better Type I error control rate (Algina \& Keselman, 1997; West, Biesanz, \& Kwok, 2004).

Item-specific PI. This type of PI typically manifests itself as a difference in RT between RN and NN trials (e.g., D'Esposito et al., 1999; Jonides et al., 1998; Nelson et al., 2003), although it sometimes also lowers accuracy (e.g., Brandon, Hirshorn, Jha, \& ThompsonSchill, 2004; Hamilton \& Martin, in press; Postle et al., 2001; Thompson-Schill et al., 2002). We calculated a normalized itemspecific PI effect on RT for each subject by first applying the formula $\left(\mathrm{RT}_{\mathrm{RN}}-\mathrm{RT}_{\mathrm{NN}}\right) / \mathrm{RT}_{\mathrm{NN}}$ to the data within each stimulus domain, in order to find the percentage change in RT that was associated with high familiarity trials, and then averaging across all domains.

\section{fMRI Data}

The principle of the fMRI time series analysis was to model the fMRI signal changes evoked by each stimulus presentation epoch (i.e., target, delay, and probe) with covariates comprising BOLD impulse response functions shifted along the timeline of the task to represent various trial epochs (Postle, Zarahn, \& D'Esposito, 2000; Zarahn, Aguirre, \& D'Esposito, 1997). The least-squares solution of the GLM of the fMRI time series data yielded parameter estimates that were associated with each covariate of interest. The smoothness of the fMRI response to neural activity allows fMRIevoked responses that arise from temporally dependent events to be resolved on the order of $4 \mathrm{sec}$ (Zarahn et al., 1997). Differences in fMRI signal (either between conditions or vs. baseline) were tested by computing $t$ statistics from contrasts between parameter estimates associated with the covariates in question. The $t$ values derived in this manner can be used as normalized indices of effect size because the residual error term that makes up the denominator of the $t$ value is positively and linearly related to the same scaling factor (or "gain effect") that characterized differences in overall BOLD signal intensity across scanning sessions (i.e., across subjects). Indeed, $t$ values may account for more unexplained intersubject variance than do percentage signal change measures (Postle et al., 2000), thereby increasing the sensitivity of random-effects group analyses.

ROIs. We divided the frontal cortex $(\mathrm{FC})$ into anatomically defined ROIs corresponding to right and left dorsolateral PFC (dlPFC), right and left ventrolateral PFC (vlPFC; left vlPFC corresponded to BA 44/47), the portion of left inferior frontal gyrus corresponding to BA 45, and dorsocaudal and ventrorostral ACC (dcACC and vrACC, respectively, corresponding to BAs 24 and 32 bilaterally). See Figure 1 for an illustration of these regions. Our rationale for including each region is as follows: Left BA 45 and left dIPFC were identified as areas sensitive to item-nonspecific PI in our previous study (Postle \& Brush, 2004). The inclusion of the remainder of left 


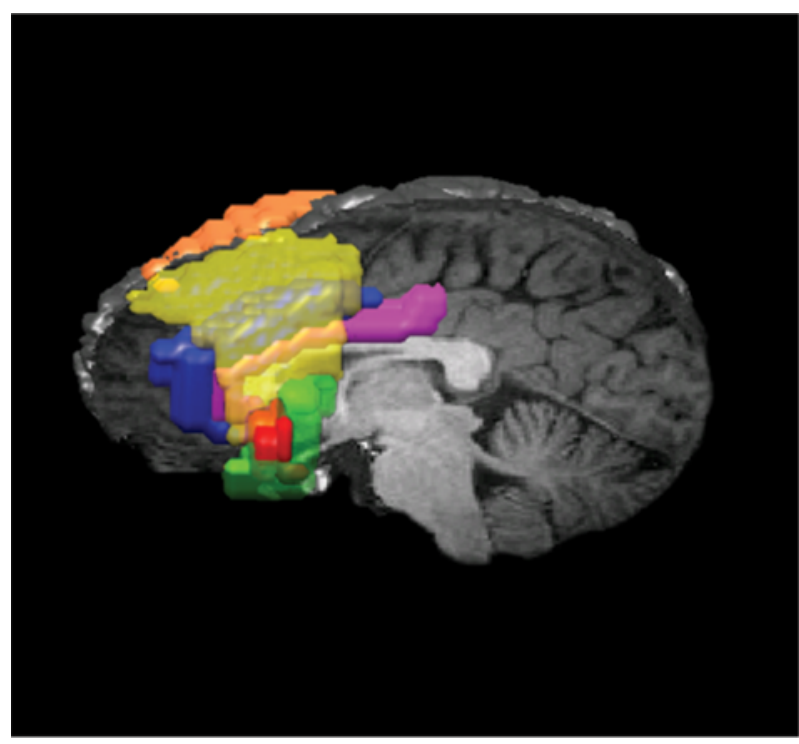

Figure 1. 3D reconstruction of the ROIs drawn for the data of Subject 35, superimposed on a 3-D reconstruction of a high resolution image of the same subject's brain. The entire left hemisphere of the anatomical image has been rendered transparent and the lateral ROIs rendered translucent, so as to display the two midline ROIs. Left dIPFC, yellow; right dIPFC, orange; left BA 44/47, green; left BA 45, red; vrACC, blue; dcACC, pink.

ventrolateral PFC (BA 44/47) and the corresponding righthemisphere regions, therefore, is important to evaluate the regional specificity of this effect. The roles of the ACC in interference resolution are complex and controversial: One group has provided evidence of a role for this area in mediating interference at the level of response selection (Nelson et al., 2003) but not with item-specific PI (Jonides et al., 2002; Nelson et al., 2003); others, on the other hand, have posited a role for the ACC with item-specific PI (Bunge, Ochsner, Desmond, Glover, \& Gabrieli, 2001; Zhang, Leung, $\&$ Johnson, 2003). Dividing this large region according one wellcharacterized functional distinction (Bush, Luu, \& Posner, 2000) offered a means of increasing our sensitivity to detect effects of interest.

The right and left dlPFC ROIs, corresponding to BAs 9 and 46 in the respective hemispheres, incorporated a portion of the superior frontal gyrus, the entire middle frontal gyrus, and an anterior portion of the inferior frontal gyrus. The right vlPFC ROI, corresponding to BAs 44,45 , and 47 , incorporated the portion of inferior frontal gyrus posterior to area 46 and anterior to the precentral sulcus. The left vlPFC (BA 44/47) ROI, defined as the pars opercularis and the inferior segment of the pars orbitalis of the inferior frontal gyrus, did not include BA 45. The left BA 45 ROI corresponded to the pars triangularis of the inferior frontal gyrus-i.e., the portion rostral to the ascending ramus and dorsal to the horizontal ramus of the sylvian fissure (Damasio, 1995). (The pars triangularis is bounded by the pars opercularis - which corresponds to BA 44-at its posterior extent and BA 46 at its anterior extent.) Finally, we defined the dcACC as the portion of the cingulate gyrus bounded at its caudal extent by the intersection of the paracingulate sulcus with the cingulate gyrus and at its rostral extent by the intersection of the callosomarginal sulcus and the cingulate sulcus, whereas the vrACC incorporated (1) the portion of the most posterior sector of the mesial surface of the superior frontal gyrus that lies caudal to the intersection of the paracingulate sulcus and the cingulate gyrus and (2) the subgenual cingulate gyrus.
Item-nonspecific PI. We assessed evidence for trial positionrelated trends in the signal attributable to the delay and the probe epochs within each of the ROIs. Based on our previous study (Postle \& Brush, 2004), we expected trial position-related trends in all FC ROIs, with the exceptions of left BA 45 and perhaps left dlPFC, to follow the trend in the global signal, which we expected to be a steady decline in epoch-evoked intensity across trials. To permit this analysis, we coded the independent variables entered into the modified GLM by epoch (target, delay, or probe) and by trial position within the block (1-16). Low-frequency drift was accounted for in the design matrix (i.e., "detrending") with scan-effect covariates. Unlike with conventional event-related analyses (Postle et al., 2000), however, we did not include trial-effect covariates, because the possible existence of offsets between trials within each scan could be the result of a tonically active PI-resolution mechanism. Thus, neither our preprocessing nor our analysis methods biased our analyses in favor of detecting either phasic or tonic effects of item-nonspecific PI. Next, we identified for each ROI all the voxels active during the trial epoch in question (e.g., with the contrast probe $\mathrm{Trial}_{1}+$ probe $_{\text {Trial } 2}+\ldots+$ probe $_{\text {Trial 16 }}$ ). Next, we determined the evoked response for the epoch in question at each trial position. (Note that with this approach, a change in $t$ value as a function of trial position could not be attributable to an increase or decrease in variance as the block progresses, because only one error term was associated with the solution of the GLM at any one voxel, and this did not change as a function of trial position.) The resultant trends would then be compared across ROIs.

Item-specific PI. This effect was assessed with a second GLM applied to the same data that coded trials as NP, NN, or RN. To test for an item-specific PI effect in the same voxels in which the itemnonspecific PI effect was evaluated, we first identified the voxels within each ROI that were reliably active during the probe epochs of all trial types (i.e., with the contrast probe $\mathrm{NP}_{\mathrm{N}}+$ probe $_{\mathrm{RN}}+$ probe $_{\mathrm{NN}}$ ) and then interrogated these voxels with the orthogonal contrast probe $_{\mathrm{RN}}-$ probe $_{\mathrm{NN}}$.

\section{RESULTS}

\section{Behavioral Results}

Item-nonspecific PI. The RT data demonstrated a pattern consistent with our prediction: an increase across the

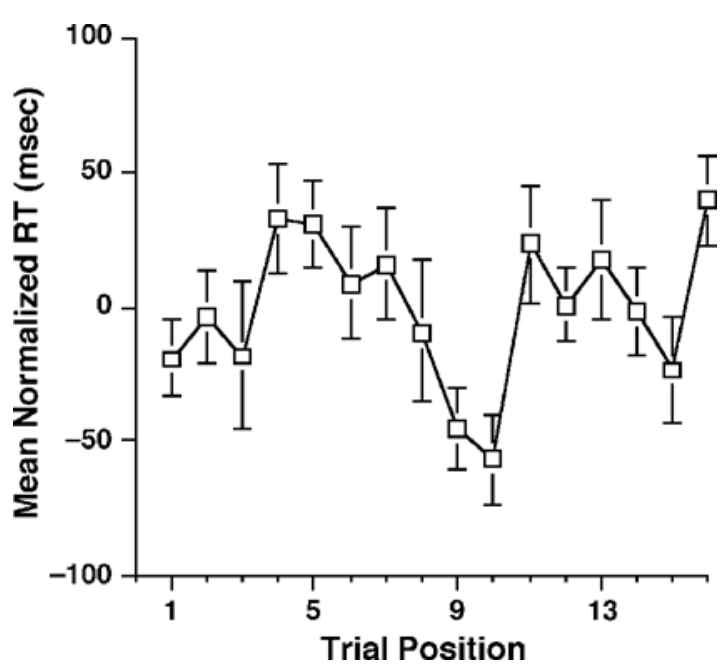

Figure 2. Group mean normalized reaction time (RT, collapsed across stimulus domain) as a function of trial position. The itemnonspecific PI effect is the increase across the first five trials. 
first several trials, followed by a noisy pattern centered around the mean RT (Figure 2). A MANOVA of the first five trials indicated that the linear trend across these trials $(y=13.8 x-36.3)$ was reliable $[F(1,24)=10.8, p<.005]$, thereby confirming the presence of an item-nonspecific PI effect. (We have previously demonstrated that a comparable trend is produced under conditions that rule out fatigue and/or flagging attention as alternative explanations of this characteristic pattern of change across time; see Postle \& Brush, 2004.)

Item-specific PI. These same subjects demonstrated a reliable mean item-specific PI effect on RT of $5.2 \%$ $[S D=6.5 ; t(24)=4.3, p<.0005$; mean RT of NN trials, $922.1 \mathrm{msec}(S D=197.8)$; of RN trials, $948.5 \mathrm{msec}$ $(S D=205.6)$; and of NP trials, $933.2 \mathrm{msec}(S D=193.7)]$. This difference was also reflected in the accuracy data [mean percentage correct $(S D)$ : NN, $90.2(6.7) ; \mathrm{RN}, 87.3$ (7.7); NP, $86.7(8.3) ; t(24)=2.4, p<.05]$.

\section{fMRI Results}

Item-nonspecific PI. The delay- and probe-evoked global signals each demonstrated a precipitous decline across the first few trials of the block and then settled into a noisy stasis centered on a level considerably lower than the signal intensity from trial 1 . The evoked response in FC ROIs tracked the trends in the global signal closely (Figure 2), although over a narrower range of intensity. The only exception to this pattern occurred in left BA 45, and only across the first two trials of the probe epoch, in which the evoked signal increased from trial 1 to trial 2. In contrast with the results from Postle and Brush (2004), a similar pattern was not observed in left dIPFC. We evaluated this effect with a repeated measures ANOVA with the factors of trial epoch (delay and probe), ROI (the seven FC ROIs), and trial position (1 and 2). This analysis revealed main effects of trial epoch $[F(1,15)=5.8, p<.05]$, ROI $[F(6,90)=3.1, p<.01]$, and trial position $[F(1,15)=6.5, p<.05]$; a marginal interaction of trial epoch and trial position $[F(1,15)=$ $4.3, p=.06$; the other 2 two-way interactions were nonsignificant $\left.\left(F_{\mathrm{S}}<1.4\right)\right]$; and a three-way interaction $[F(6,90)=2.2, p=.05]$. (Note that missing values in one or more cells meant that data from 9 subjects were excluded from this ANOVA.) Follow-up contrasts that compared the trial 1 to trial 2 difference in left BA 45 with the same difference aggregated across all other FC ROIs confirmed that the trend in left BA 45 differed from the trend in the rest of FC in the probe epoch $[t(22)=$ $2.3, p<.05]$ but not the delay epoch $[t(20)=0.7$, n.s. $]$. Additionally, the trial 1 to trial 2 difference in left BA 45 differed from its analogue in the global signal in the probe epoch $[t(22)=4.6, p<.0005]$ but not in the delay epoch $[t(20)=1.4$, n.s. $]$.

One possible concern with the interpretation of these results as evidence for an item-nonspecific PI effect is that the two sources of PI were confounded in many of the initial trials of this study. That is, the analyses presented in the preceding paragraph included blocks in which the second trial featured an $\mathrm{RN}$ probe. In such a case, one would expect a trial 1 to trial 2 increase in probe-evoked response in left BA 45 simply due to the presence of item-specific PI in the second, but not the first, trial. To address this concern, we repeated the analyses as described in the preceding paragraph, but limited them to the probe epochs from blocks in which no RN probes appeared in the first two trials. This new analysis included 9 blocks out of the total of 21 ( 7 blocks/experiment $\times 3$ forms). Each of the stimulus domains except color was represented among these 9 . The repeated measures ANOVA of this new data set, with the factors ROI (the seven FC ROIs) and trial position ( 1 and 2 ), revealed a main effect of ROI $[F(6,138)=5.8, p<.0005]$, no effect of trial position $[F(1,23)=2.1$, n.s.], and an interaction $[F(6,138)=2.3, p<.05]$. Follow-up contrasts once again confirmed that the trial 1 to trial 2 trend in probe-evoked signal differed between left BA 45 and all other FC ROIs $[t(24)=2.0, p=.05]$, as well as between left BA 45 and the global signal $[t(24)=4.1, p<.0005]$. See Figure 3 for a display of these results.

Item-specific PI. Tests for an item-specific PI effect within voxels active during the probe epoch of our task failed to find a reliable effect in any FC ROI, although the effect in left BA 45 approached significance $[t(24)=$ $1.9, p=.07]$. Additionally, the item-specific PI effect in this ROI was significantly different from the mean effect aggregated across the other FC ROIs $[t(23)=3.2, p<$ .005 ; see Table 1]. So modest an item-specific PI effect in left BA 45 was unexpected, because the association of item-specific PI with activity in left BA 45 is robust and has been replicated many times by many different research groups (e.g., Burgess \& Braver, 2004; D’Esposito et al., 1999; Derrfuss, Brass, \& von Cramon, 2004; Hedden \& Yoon, 2004; Jonides et al., 1998; Martin, Hamilton, Lipszyc, \& Potts, 2004; Nelson et al., 2003; Postle et al., 2001). One explanation might be the heterogeneity of stimulus domains across which the item-specific PI effect was computed. Table 2 presents the behavioral correlates of item-specific PI broken out by stimulus domain and suggests that the effect was carried, to varying degrees, by five of them: digits, faces, letters, locations, and words. When restricted to these five stimulus domains, the aggregate RT item-specific PI effect increased to $7.4 \%$ $[S D=6.6 ; t(28)=5.9, p<.0001$; mean RT of NN trials, $918.1 \mathrm{msec}(S D=184.0)$; of RN trials, $976.8 \mathrm{msec}$ $(S D=196.7)]$. The corresponding item-specific PI effect in BA 45, however, did not achieve significance $[t(24)=1.6$, n.s. $]$.

\section{Individual Differences}

Despite the fact that our results did not yield an unequivocally strong effect in BA 45 , we though it worth investigating evidence for brain-behavior correlations in the item-specific PI effect. The results, incorporating all domains, indicated a nonsignificant negative relation between the RT correlate of item-specific PI and its fMRI correlate in left BA $45(r=-.26 ; p=.22)$. 


\section{A}

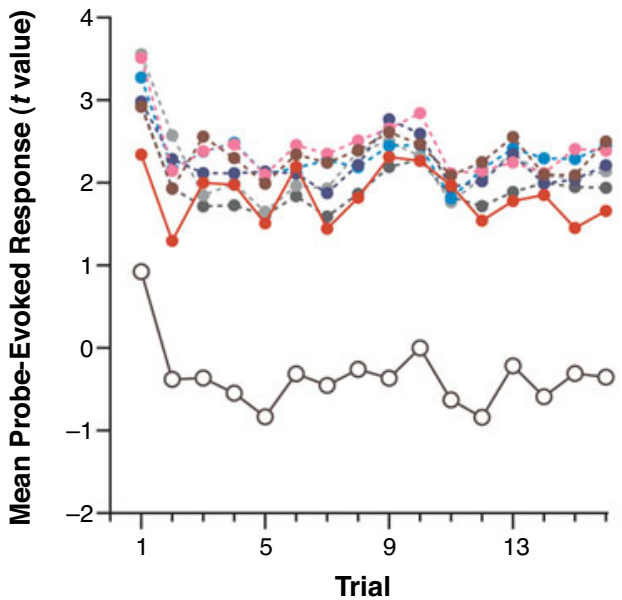

ROI

B

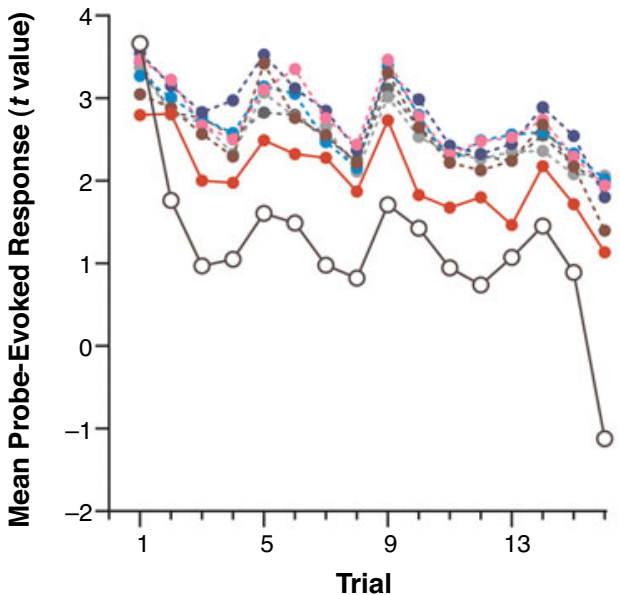

ROI

C

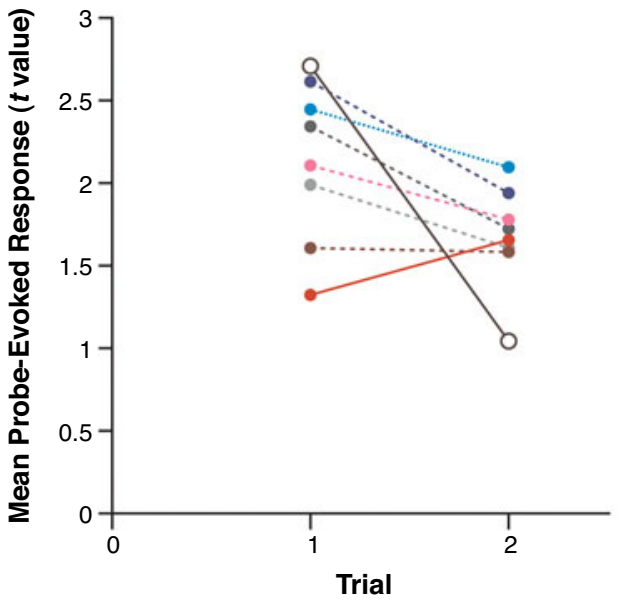

\section{ROI}

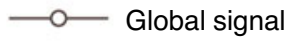

$\longrightarrow$ Left BA 45

........ Left vIPFC

Left dIPFC

Right vIPFC

Right dIPFC

vrACC

dcACC

Figure 3. (A) Item-nonspecific PI analyses: Mean probe-evoked response as a function of trial position, by ROI. (B) Item-nonspecific PI analyses: Mean probe-evoked response as a function of trial position, by ROI. (C) Itemnonspecific PI analyses restricted to blocks in which there were no RN probes in the first two trials: Mean probe-evoked response as a function of trial position, by ROI. 
Table 1

Mean fMRI Item-Specific PI Effect $\left(\right.$ probe $_{\mathrm{RN}}-$ probe $\left._{\mathrm{NN}}\right)$ From Voxels Identified With the Contrast $\left(\right.$ probe $_{\mathrm{NP}}+$ probe $_{\mathrm{RN}}+$ probe $\left._{\mathrm{NN}}\right)$, by ROI

\begin{tabular}{lrrrcccr}
\hline & Right & Left & Right & Left & Left & & \\
& dlPFC & dlPFC & vlPFC & BA 44/47 & BA 45 & vrACC & dcACC \\
\hline$t$ & -.34 & -.32 & -.11 & .02 & .33 & -.41 & -.20 \\
$S E$ & .19 & .19 & .22 & .22 & .21 & .21 & .21 \\
\hline
\end{tabular}

\section{DISCUSSION}

The results of our experiment confirmed our prediction that left BA 45 would demonstrate sensitivity to both item-specific and nonspecific PI. They are thus consistent with the hypothesis that common processes may underlie the mediation of these two types of PI. One possible result of this hypothesis might be to bridge the literatures on these two phenomena that have, until now, proceeded independently of each other. One of these phenomena, item-specific PI, has been well characterized neurophysiologically, but its theoretical implications have only recently begun to garner attention (e.g., Brandon et al., 2004; Braver et al., in press; ThompsonSchill et al., 2002). The other, item-nonspecific PI, has a rich theoretical basis (Hasher \& Zacks, 1988; Keppel \& Underwood, 1962; May et al., 1999; Wickens et al., 1963), but little is known about its instantiation in the brain. Establishing that a common process or set of processes underlies the mediation of the two could lead to increased understanding of both and, more generally, of the organization of PFC-based control processes.

With respect to item-nonspecific PI and its mediation, the present study confirms two facts that were unresolved in Postle and Brush (2004): The effect is localized in time to the probe epoch and in space to left BA 45. The implication of the former finding is that, as with itemspecific PI, the putative mechanism mediating the effects of item-nonspecific PI operates in a phasic manner, time-locked to the onset of the probe. Our method lacks the sensitivity, however, to distinguish between, for example, a mechanism contributing to probe evaluation or one responsible for discarding items upon the completion of the trial. With regard to neuroanatomical localization, Postle and Brush identified left BA 45 and left dlPFC as differentially sensitive to item-nonspecific PI and suggested that the predictability of PI (highly pre- dictable for item-nonspecific, unpredictable for itemspecific) may determine the spatial extent of its neural correlates. The results of the present study, however, do not support this idea. Finally, and unexpectedly, the fMRI correlate of item-nonspecific PI was only observed across two trials, whereas the behavioral effect persisted across five. Understanding the factors relating the behavioral and neural correlates of item-nonspecific PI, therefore, will be an important goal for future research.

We turn now to item-specific PI. When we designed our study with seven different domains of stimuli, we did so with the assumption that item-specific PI is mediated by a domain-general control process (see also Brandon et al., 2004), and therefore that collapsing across stimulus domains would not compromise our ability to produce an item-specific PI effect. In retrospect, however, we realize that this assumption requires careful empirical vetting. We know of two other studies of item-specific PI that have used stimuli other than words or letters. One study employed faces as stimuli and found sensitivity to $\mathrm{RN}$ probes in inferior frontal gyrus in both the left and right hemispheres (see also Brandon et al., 2004). A second study, requiring memory for locations and employing a within-trial directed forgetting procedure, found sensitivity to RN probes in precentral sulcus and superior parietal lobule of the right hemisphere, but not in BA 45 in either hemisphere (Leung \& Zhang, 2004). In the data presented in this report, both the behavioral and the fMRI correlates of item-specific PI varied across stimulus domains. Behaviorally, two stimulus domains, colors and shapes, did not produce item-specific PI effects. At face value, this result is surprising, because we know of no reason to expect a priori that mnemonic processing of stimuli of any particular type would not interfere with memory for subsequent presentations of stimuli drawn from the same domain of information. One possible explanation of this aspect of our results is that differences in verbalizability explain the observed variability across domains. Consistent with this idea are results from Hamilton and Martin (in press), who describe a patient with damage to the left inferior PFC who is selectively impaired on tests of verbal, but not nonverbal, inhibition. We find the verbalizability explanation unsatisfactory for our results, however, because we and others have shown that a verbal code is invariably recruited for working and short-term memory for shapes (Postle, D’Esposito, \& Corkin, in press; Simons, 1996)

Table 2

Mean Behavioral Item-Specific PI Effects, by Stimulus Domain

\begin{tabular}{|c|c|c|c|c|c|c|c|}
\hline & Color & Digits & Faces & Letters & Locations & Shapes & Words \\
\hline Percentage of RT ${ }^{\mathrm{a}}$ & -0.8 & 3.9 & 4.5 & $12.3 *$ & 4.8 & 0.0 & $11.9 *$ \\
\hline$S E$ & 5.0 & 2.9 & 3.6 & 2.8 & 4.0 & 3.4 & 4.7 \\
\hline Accuracy (in percentage correct ${ }^{b}$ ) & $15.6^{*}$ & -0.4 & -2.0 & $-11.1 *$ & $-7.3^{*}$ & $5.8^{*}$ & $-9.3^{*}$ \\
\hline$S E$ & 5.9 & 1.6 & 3.3 & 2.9 & 3.7 & 2.5 & 5.4 \\
\hline
\end{tabular}

*Effects that are significantly different from 0 at $p<.05 . \quad{ }^{a}$ Calculated $\left(\mathrm{RT}_{\mathrm{RN}}-\mathrm{RT}_{\mathrm{NN}}\right) / \mathrm{RT}_{\mathrm{RN}} . \quad{ }^{\mathrm{b}}$ Calculated accuracy $_{\mathrm{RN}}-\operatorname{accuracy}_{\mathrm{NN}}$; note that negative values in the accuracy data correspond to accuracy $\mathrm{NN}_{\mathrm{NN}}>\operatorname{accuracy}_{\mathrm{RN}}$. 
and colors (Postle, Messner, \& Cappa, 2004), but not for locations (Postle et al., in press; Postle, Messner, \& Cappa, 2004; Simons, 1996). A second possibility is that the absence of "recent positive" probes from our design created a situation in which the familiarity signal generated by PI-producing lures on RN trials may have, paradoxically, provided a source of evidence that $\mathrm{RN}$ probes were, indeed, negative. This could happen, goes the reasoning, because positive probes were never accompanied by this same "recent" familiarity signal. A third explanation of the variability across stimulus domains of the item-specific PI effect is that having only five $\mathrm{RN}$ and four NN trials per domain per subject may simply not have been enough to produce a robust item-specific PI effect. Consistent with this interpretation is the fact that of the five domains for which the effect was in the predicted direction, the effect only achieved significance in the RT results for two domains, and in the accuracy results for three domains. Thus, perhaps these complex effects of stimulus domain contributed to the weaker-thanexpected item-specific PI effect in the fMRI data. The same reasoning may also be relevant to consideration of the equivocal result from the individual differences analysis. Also, the possibility that the application of proactive versus reactive control (Braver et al., in press; Reynolds et al., 2004) is mediated by individual differences in working memory capacity (Burgess \& Braver, 2004) may have worked against us here, for we did not measure that factor in this study. (In this framework, the item-specific PI effects referred to throughout this paper would be related to reactive control.)

Thus, at the conclusion of this study, we find ourselves in a position that is all too familiar to many scientists. On one hand, our results support the common processes hypothesis that motivated the study. On the other, they have also brought to our awareness a new factor that may require reevaluation of an assumption underlying the hypothesis itself. It is fitting for this special issue that the new factor is that of domain specificity of the putative PFC-based control system(s), an issue that was a focus of Patricia Goldman-Rakic's research for much of her career.

\section{REFERENCES}

Aguirre, G. K., Zarahn, E., \& D'Esposito, M. (1998). The variability of human, BOLD hemodynamic responses. NeuroImage, 8,360 369.

Algina, J., \& Keselman, H. J. (1997). Detecting repeated measures effects with univariate and multivariate statistics. Psychological Methods, 2, 208-218.

Attneave, F., \& Arnoult, M. D. (1956). The quantitative study of shape and pattern perception. Psychological Bulletin, 53, 452-471.

Battig, W. F., \& Montague, W. E. (1969). Category norms for verbal items in 56 categories: A replication and extension of the Connecticut category names. Journal of Experimental Psychology Monographs, $\mathbf{8 0}(3$, Pt. 2).

Boynton, G. M., Engel, S. A., Glover, G. H., \& Heeger, D. J. (1996). Linear systems analysis of functional magnetic resonance imaging in human V1. Journal of Neuroscience, 16, 4207-4221.

Brandon, M., HirShorn, E. A., JHA, A. P., \& THOMPSON-Schill, S. L.
(2004, April). Proactive interference resolution during nonverbal working memory: Evidence for domain-general processing in LIFG. Poster presented at the Cognitive Neuroscience Society Annual Meeting, San Francisco.

Braver, T. S., Gray, J. R., \& Burgess, G. C. (in press). Explaining the many varieties of working memory variation: Dual mechanisms of cognitive control. In A. Conway, C. Jarrold, M. Kane, A. Miyake, \& J. Towse (Eds.), Variation in working memory. Oxford: Oxford University Press.

Bunge, S. A., Ochsner, K. N., Desmond, J. E., Glover, G. H., \& Gabrieli, J. D. E. (2001). Prefrontal regions involved in keeping information in and out of mind. Brain, 124, 2074-2086.

Burgess, G. C., \& Braver, T. S. (2004, April). Dual mechanisms of cognitive control over interference. Poster presented at the Cognitive Neuroscience Society Annual Meeting, San Francisco.

Bush, G., LuU, P., \& Posner, M. I. (2000). Cognitive and emotional influences in anterior cingulate cortex. Trends in Cognitive Sciences, 4 , 215-222.

DAMASIO, H. (1995). Human brain anatomy in computerized images. Oxford: Oxford University Press.

Derrfuss, J., Brass, M., \& von Cramon, Y. (2004, April). Cognitive control in the posterior frontolateral cortex: Evidence from common activations in task coordination, interference control, and working memory. Poster presented at the Cognitive Neuroscience Society Annual Meeting, San Francisco.

D’Esposito, M., Postle, B. R., Jonides, J., \& Smith, E. E. (1999). The neural substrate and temporal dynamics of interference effects in working memory as revealed by event-related functional MRI. Proceedings of the National Academy of Sciences, 96, 7514-7519.

Friston, K. J., Holmes, A. P., Poline, J.-B., Heather, J. D., \& FrackOWIAK, R. S. J. (1995). Analysis of fMRI time-series revisited. NeuroImage, 2, 45-53.

GoldMAN-RaKIC, P. S. (1992). Working memory and the mind. Scientific American, 267, 110-117.

Hamilton, A. C., \& Martin, R. C. (in press). Dissociations among tasks involving inhibition: A single-case study. Cognitive, Affective, \& Behavioral Neuroscience.

Handwerker, D. A., Ollinger, J. M., \& D'Esposito, M. (2004). Variation of BOLD hemodynamic responses across subjects and brain regions and their effects on statistical analyses. NeuroImage, 21, 16391651.

HASHer, L., \& ZACKs, R. T. (1988). Working memory, comprehension, and aging: A review and a new view. In G. H. Bower (Ed.), The psychology of learning and motivation (Vol. 22, pp. 193-225). San Diego: Academic Press.

HedDEN, T., \& Yoon, C. (2004, April). Neuropsychological measures of distinct executive functions predict interference in short-term memory for older adults [Abstract]. Cognitive Neuroscience Society Annual Meeting Program 2004, 164.

Jonides, J., Badre, D., Curtis, C., Thompson-Schill, S., \& Smith, E. E. (2002). Mechanisms of conflict resolution in prefrontal cortex. In D. T. Stuss \& R. T. Knight (Eds.), Principles of frontal lobe function (pp. 233-245). Oxford: Oxford University Press.

Jonides, J., Marshuetz, C., Smith, E. E., Reuter-Lorenz, P. A., \& KoEPPE, R. A. (2000). Age differences in behavior and PET activation reveal differences in interference resolution in verbal working memory. Journal of Cognitive Neuroscience, 12, 188-196.

Jonides, J., Smith, E. E., Marshuetz, C., Koeppe, R. A., \& ReuterLORENZ, P. A. (1998). Inhibition of verbal working memory revealed by brain activation. Proceedings of the National Academy of Sciences, 95, 8410-8413.

JoSEPHS, O., TURNER, R., \& Friston, K. (1997). Event-related fMRI. Human Brain Mapping, 5, 243-248.

KEPPEL, G., \& UNDERWOOD, B. J. (1962). Proactive inhibition in shortterm retention of single items. Journal of Verbal Learning \& Verbal Behavior, 1, 153-161.

KNight, R. T., Staines, W. R., Swick, D., \& Chao, L. L. (1999). Prefrontal cortex regulates inhibition and excitation in distributed neural networks. Acta Psychologica, 101, 159-178.

Kwong, K. K., Belliveau, J. W., Chesler, D. A., Goldberg, I. E., 
Weisskoff, R. M., Poncelet, B. P., Kennedy, D. N., Hoppel, B. E., Cohen, M. S., Turner, R., Cheng, H.-M., Brady, T. J., \& Rosen, B. R. (1992). Dynamic magnetic resonance imaging of human brain activity during primary sensory stimulation. Proceedings of the $\mathrm{Na}$ tional Academy of Sciences, 89, 5675-5679.

LEUNG, H.-C., \& ZHANG, J. X. (2004). Interference resolution in spatial working memory. NeuroImage, 23, 1013-1019.

Lustig, C., MAY, C. P., \& Hasher, L. (2001). Working memory span and the role of proactive interference. Journal of Experimental Psychology: General, 130, 199-207.

Martin, R. C., Hamilton, A. C., Lipszyc, M., \& Potts, G. F. (2004, April). Manipulation of inhibition demands in a working memory task: Evidence from patient and ERP data. Poster presented at the Cognitive Neuroscience Society Annual Meeting, San Francisco.

MAY, C. P., HASHER, L., \& KANE, M. J. (1999). The role of interference in memory span. Memory \& Cognition, 27, 759-767.

Monsell, S. (1978). Recency, immediate recognition memory, and reaction time. Cognitive Psychology, 10, 465-501.

Nelson, J. K., Reuter-Lorenz, P. A., Sylvester, C. Y. C., Jonides, J., \& SMith, E. E. (2003). Dissociable neural mechanisms underlying response-based and familiarity-based conflict in working memory. Proceedings of the National Academy of Sciences, 100, 11171-11175.

Ogawa, S., Tank, D. W., Menon, R., Ellermann, J. M., Kim, S. G., Merkle, H., \& Ugurbil, K. (1992). Intrinsic signal changes accompanying sensory stimulation: Functional brain mapping with magnetic resonance imaging. Proceedings of the National Academy of Sciences, 89, 5951-5955.

Postle, B. R., Awh, E., Jonides, J., Smith, E. E., \& D’Esposito, M. (2004). The where and how of attention-based rehearsal in spatial working memory. Cognitive Brain Research, 20, 194-205.

Postle, B. R., Berger, J. S., \& D'Esposito, M. (1999). Functional neuroanatomical double dissociation of mnemonic and executive control processes contributing to working memory performance. Proceedings of the National Academy of Sciences, 96, 12959-12964.

Postle, B. R., Berger, J. S., Goldstein, J. H., Curtis, C. E., \& D'Esposito, M. (2001). Behavioral and neurophysiological correlates of episodic coding, proactive interference, and list length effects in a running span verbal working memory task. Cognitive, Affective, \& Behavioral Neuroscience, 1, 10-21.

PostLE, B. R., \& BRUSH, L. N. (2004). The neural bases of the effects of item-nonspecific proactive interference in working memory. $\mathrm{Cog}$ nitive, Affective, \& Behavioral Neuroscience, 4, 379-392.

Postle, B. R., \& D'EsPosito, M. (1999). "What"-then-"where" in visual working memory: An event-related fMRI study. Journal of Cognitive Neuroscience, 11, 585-597.

PostLe, B. R., \& D'Esposito, M. (2003). Spatial working memory activity of the caudate nucleus is sensitive to frame of reference. $\mathrm{Cog}$ nitive, Affective, \& Behavioral Neuroscience, 3, 133-144.

Postle, B. R., D’EsPosito, M., \& CoRKIn, S. (in press). Effects of verbal and nonverbal interference on spatial and object visual working memory. Memory \& Cognition.

Postle, B. R., Druzgal, T. J., \& D'Esposito, M. (2003). Seeking the neural substrates of working memory storage. Cortex, 39, 927-946.
Postle, B. R., Messner, M., \& CAPPA, S. (2004). Verbal contributions to short-term retention of color information. Manuscript submitted for publication.

Postle, B. R., Zarahn, E., \& D’Esposito, M. (2000). Using eventrelated $\mathrm{fMRI}$ to assess delay-period activity during performance of spatial and nonspatial working memory tasks. Brain Research Protocols, 5, 57-66.

ReYNolds, J. R., Donaldson, D. I., Wagner, A. D., \& Braver, T. S. (2004). Item- and task-level processes in the left inferior prefrontal cortex: Positive and negative correlates of encoding. NeuroImage, 21, $1472-1483$

SiMONS, D. J. (1996). In sight, out of mind: When object representations fail. Psychological Science, 7, 301-305.

Thompson-Schill, S. L., Jonides, J., Marshuetz, C., Smith, E. E., D'Esposito, M., Kan, I. P., KNIGHT, R. T., \& Swick, D. (2002). Effects of frontal lobe damage on interference effects in working memory. Cognitive, Affective, \& Behavioral Neuroscience, 2, 109-120.

VANDERPLAS, J. M., \& Garvin, E. A. (1959). The association value of random shapes. Journal of Experimental Psychology, 57, 147-154.

West, S. G., Biesanz, J. C., \& KwoK, O.-M. (2004). Within-subject and longitudinal experiments: Design and analysis issues. In C. Sansone, C. C. Morf, \& A. T. Panter (Eds.), The Sage handbook of methods in social psychology (pp. 287-312). Thousand Oaks, CA: Sage. WiCKens, D. D. (1973). Some characteristics of word encoding. Memory \& Cognition, 1, 485-490.

Wickens, D. D., Born, D. G., \& Allen, C. K. (1963). Proactive inhibition and item similarity in short-term memory. Journal of Verbal Learning \& Verbal Behavior, 2, 440-445.

WORSLEY, K. J., \& FrISTON, K. J. (1995). Analysis of fMRI time-series revisited-again. NeuroImage, 2, 173-182.

Zarahn, E., Aguirre, G. K., \& D’EsPosito, M. (1997). A trial-based experimental design for fMRI. NeuroImage, 6, 122-138.

Zhang, J. X., Leung, H.-C., \& JoHnson, M. K. (2003). Frontal activations associated with accessing and evaluating information in working memory: An fMRI study. NeuroImage, 20, 1531-1539.

\section{NOTES}

1. This rationale is only valid for two cognitive phenomena that could plausibly be supported by different mechanisms. Thus, it does not apply, for example, when visual stimulation in the upper left versus the lower right quadrants of the visual field activates different areas of calcarine cortex.

2. The flower names were lily, carnation, lilac, pansy, peony, petunia, gardenia, dandelion, geranium, tulip, daisy, daffodil, violet, azalea, orchid, and iris. We excluded rose, even though it occurs in the top 16 in the Battig and Montague (1969) norms, because we judged it to be disproportionately salient.

(Manuscript received July 26, 2004; revision accepted for publication December 1, 2004.) 